Rev. Elev. Méd. vét. Pays trop., 1970, 23 (4) : 479-91

\title{
Théorie et pratique des mesures de la pression osmotique par cryométrie
}

\author{
par J.P. PETIT $\left({ }^{*}\right)$
}

\begin{abstract}
RESUME
Lors des études de l'hémolymphe des glossines la première détermination a été celle de la pression osmotique. L'étude des sérums animaux n'échappe pas à cette étape analytique et la connaissance des solutions salines utilisées en physiopathologie passe également par la détermination des abaissements de point de congélation. Et toujours des questions se posent à propos du calcul de la pression osmotique dont l'importance en biologie et dont la variété des formulations laissaient la place à une mise au point reposant sur des bases physiques solides.

Après avoir repris les définitions, la partie théorique de l'exposé permet de passer en revue à la fois les lois relatives aux masses moléculaires dans le cas général, aussi bien que dans les cas particuliers, et les lois relatives aux pressions osmotiques. On aboutit ainsi logiquement à une série de formules qui en sont l'aboutissement et qui permettent d'envisager des applications pratiques, principalement le calcul de la pression osmotique $\pi$ en mesurant l'abaissement $\Delta$ du point de congélation d'une solution aqueuse. On a pris pour valeur des constantes utilisées et pour unités, celles retenues le plus récemment par les accords internationaux. Tout au long de l'établissement des formules, leurs conditions de validité sont longuement soulignées, puis ces précautions élémentaires étant prises, des applications sont étudiées du point de vue pratique.

En particulier, la manipulation la plus simple pour déterminer un $\Delta$ est exposée, ayec pour appareillage, un cryomètre de type Beckmann à thermomètre différentiel. La technique est décrite pas à pas, ce qui permet de refaire une mesure avec ces seules indications. Les autres techniques plus complexes ou plus coûteuses sont seulement mentionnées.

Quelques résultats illustrent l'ensemble, ils concernent des plasmas de bovins africains et des hémolymphes de glossines. Enfin un tableau rassemble les $\Delta$ de quelques substances d'un emploi courant au laboratoire, ces données ayant été calculées ou mesurées peuvent éviter d'avoir recours à une mesure pour laquelle on n'est pas toujours équipé.

L'ensemble des symboles utilisés et leur signifıcation sont présentés à la fin du texte pour l'alléger et éviter les répétitions.
\end{abstract}

\section{INTRODUCTION}

La pression osmotique a un rôle fondamental en biologie où elle permet, sur le plan théorique, de comprendre de nombreux mécanismes de fonctionnement des êtres vivants, sur le plan pratique, de réaliser des solutions isotoniques aux divers liquides biologiques dans des buts variés : par exemple l'étude des conditions phy-

(*) I.E.M.V.T. Service de Biochimie. sico-chimiques des cultures microbiennes ou cellulaires. Pourtant, sa détermination ou son calcul théorique sont pratiqués avec des formules très diverses et nécessitent des constantes dont la recherche aboutit à des valeurs différentes, ce qui est pour le moins paradoxal. C'est pourquoi il est apparu nécessaire de tenter une mise au point concernant cette importante notion, qui demeure partiellement obscure quand on cherche à l'aborder sous son double aspect théorique et pratique. 
Le phénomène physique lui-même est potentiel et pour le mettre en évidence il faut recourir à un artifice révélé par l'expérience classique de Dutrochet (figure 1): une membrane perméable, c'est-à-dire laissant passer librement le solvant (SV) mais plus lentement Je corps dissous (soluté $\mathrm{ST}$ ), sépare au temps 1 de l'expérience le solvant de la solution. A l'équilibre (temps 2), quand le liquide est monté à la hauteur maximale ( $\Delta \mathrm{HT}$ ) dans le tube capillaire, la pression hydrostatique $\Delta \mathrm{HT}$ équilibre la pression osmotique qui règne au sein de la solution $\mathrm{SN}[\mathrm{SN}=$ solvant $(\mathrm{SV})+$ soluté (ST)], la différence des niveaux en permet une évaluation difficile, car la substance dissoute diffuse lentement et les forces osmotiques qui agissent de part et d'autre de la membrane finjssent par s'équilibrer. Cette expérience a seulement montré l'existence de la pression osmotique. C'est Pfeiffer (figure 2) qui a su réaliser le premier une véritable membrane semi-perméable arrêtant le soluté mais laissant passer librement le solvant, ici de l'eau. Elle monte dans le tube et ne redescend plus, on a ainsi un moyen précis de mesurer la pression osmotique. Ce principe est appliqué dans la chimie macromoléculaire pour l'étude des substances colloïdales. S'il existe en effet de nombreux procédés pour réaliser des membranes semi-perméables vis-à-vis des grosses molécules, il est par contre extrêmement délicat d'y parvenir pour des corps à poids moléculaire faible. Les propriétés sélectives des membranes fabriquées par les êtres vivants ne sont que grossièrement simulées par ces types de membranes.

Il existe plusieurs procédés, qui permettent des mesures directes de la pression osmotique $\pi$ (appareil de Hellfritz, de Zimm-Myerson, d'Elias et Ritscher, pour ne citer que ceux qui sont commercialisés). Mais ils peuvent difficilement entrer dans la pratique courante des laboratoires de biologie à cause de la délicatesse tant de la fabrication des membranes que de l'exécution des mesures et des délais nécessaires (plusieurs jours). Ils servent surtout à la détermination approchée des masses moléculaires $\mathrm{M}$ des composés macromoléculaires.

Le procédé le plus pratique et le moins coûteux actuellement consiste à effectuer une mesure indirecte par détermination de l'abaissement $\Delta$ du point de congélation d'une solution comparée à celle du solvant pur: il s'agit de

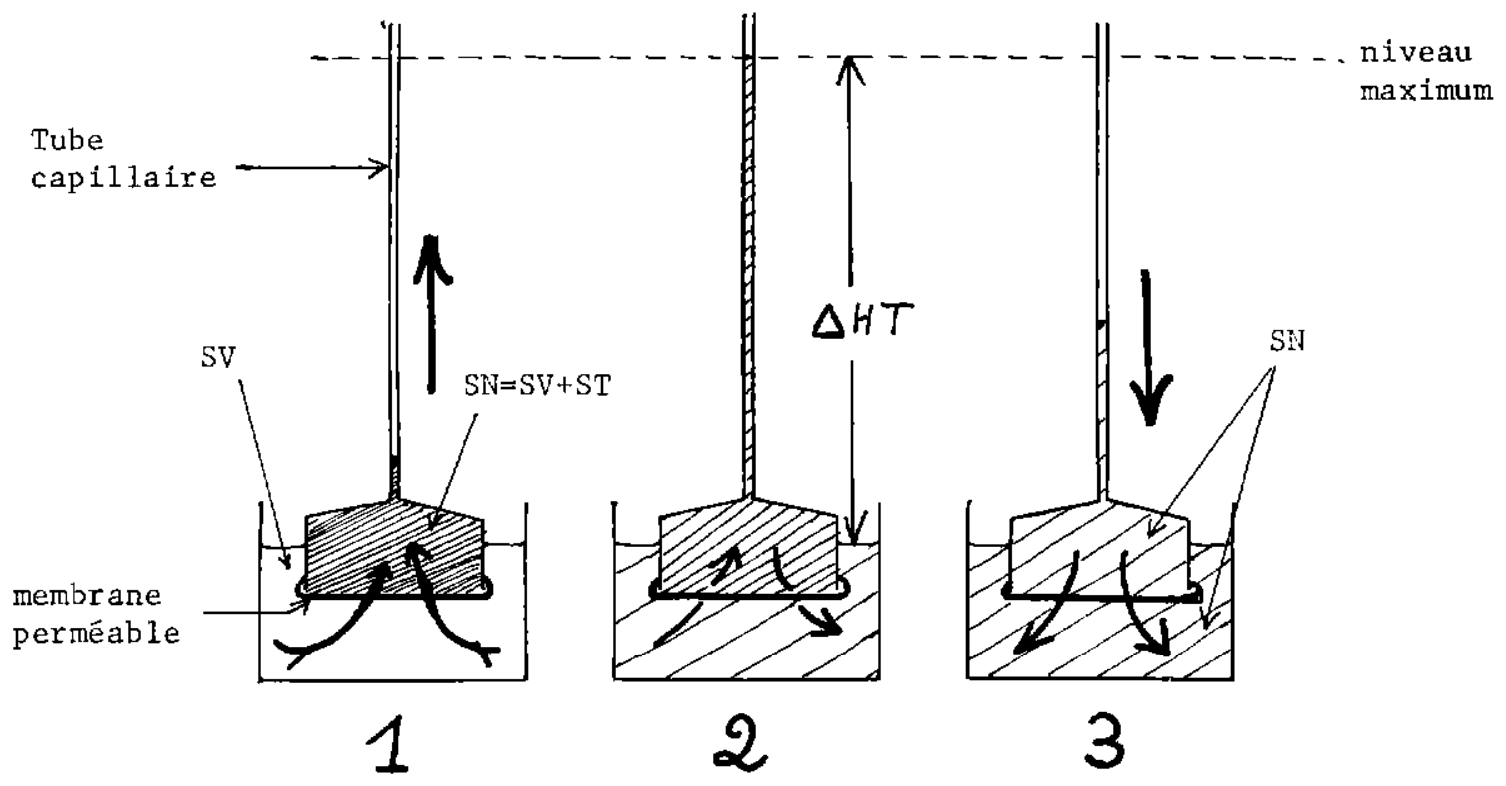

Fig. 1.

Expérience de Dutrochet: les flèches montantes indiquent le sens de déplacement du solvant (SV), pour le soluté (ST) elles descendent. 


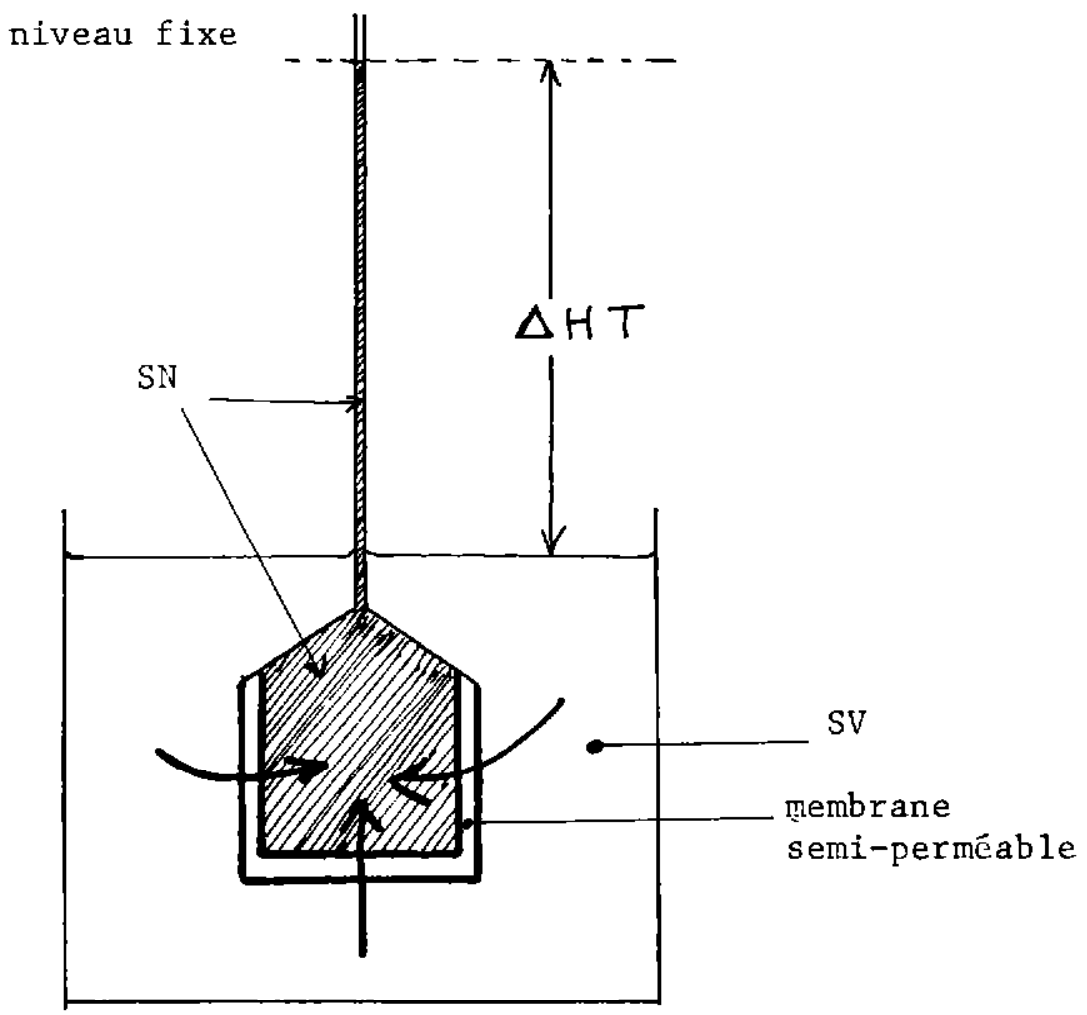

Fig. 2.

Expérience de Pfeiffer: une fois le niveau le plus haut atteint dans le tube capillaire, il n'y a plus de variations dues à la diffusion du Soluté (ST).

la cryométrie. $\Delta$ est lié par une formule simple à $\pi$.

Dans un but pratique et à cause de la grande diversité des présentations de ce sujet, nous envisagerons les lois fondamentales sur lesquelles on doit s'appuyer, en insistant sur le mode de calcul de $\pi$, selon que l'on a affaire à des solutés dissociés ou non: ces principes seront appliqués de façon pratique pour mesurer $\Delta$ dans le cas des liquides biologiques et des solutions salines simples. L'ensemble, sans chercher à être complet, essaie d'apporter une certaine clarté sur ce sujet.

\section{LOIS RELATIVES AUX MASSES MOLECULAIRES}

L'utilisation de divers symboles étant indispensable à la clarté de l'exposé, ils seront définis au fur et à mesure de leur apparition et leur ensemble est regroupé à la fin de l'article par ordre alphabétique.

\section{Loi de Blagden}

Elle souligne le fait qu'une solution saline se congèle à une température inférieure à celle du solvant et que l'abaissement de ce point de congélation est à peu près proportionnel à la concentration.

\section{Loi de Raoult}

Il s'agit d'une généralisation de la loi de Blagden aux substances organiques.

\section{Enoncé}

L'abaissement du point de congélation d'une solution étendue et non électrolyte est (on ne doit pas oublier ces termes dans l'énoncé exact de la loi) :

$1^{o}$ proportionnel à sa concentration; 
$2^{\circ}$ inversement proportionnel à la masse moléculaire du corps dissous.

Notons que la mise en solution ne doit pas provoquer de condensation moléculaire ni de décomposition pour que la loi puisse s'appliquer; en fait, c'est une loi limite.

$$
\Delta=\mathrm{k} \frac{\mathrm{C}}{\mathrm{M}}
$$

$$
\begin{array}{ll}
\Delta & : \text { voir } 2, a ; \\
\mathrm{k} & : \text { voir } 2, c ; \\
\mathrm{C} \text { et } \mathrm{M}: & \text { voir } 2, b .
\end{array}
$$

\section{Paramètres}

a) Abaissement du point de congélation : $\Delta$

$$
\Delta=\mathbf{t}_{\mathbf{v}}-\mathbf{t}
$$

$\mathrm{t}_{\mathrm{a}}$ : point de congélation du solvant pur (SV) en degré $\mathrm{C}$;

$\mathrm{t}$ : point de congélation de la solution $(\mathrm{SV}+$ ST) en degré $C$.

Remarquons bien que $\Delta$, abaissement du point de congélation est positif et s'exprime en degré $\mathrm{C}$.

Exemple : pour un sérum, on a

d'où

$$
t_{0}=0^{\circ} \mathrm{C} \text { et } \mathrm{t}=-0,56^{\circ} \mathrm{C}
$$

$$
\Delta=0-(-0,56)=0,56^{\circ} \mathrm{C}
$$

b) Concentration du corps dissous : C

$$
\mathrm{C}=\frac{\mathrm{m}}{\mathrm{m}+\mathrm{m}^{\prime}}
$$

C : concentration du soluté dans la solution;

m : masse du corps dissous;

$\mathrm{m}^{\prime} \quad$ : masse du solvant exprimée dans la même unité que $\mathbf{m}$;

$\mathrm{m}+\mathrm{m}^{\prime}$ : masse totale de la solution.

$\mathrm{Au}$ dénominateur, $m$ est négligeable par rapport à $\mathrm{m}^{\prime}$, puisque la solution est supposée étendue.

$$
\text { Donc } \quad \mathrm{C}=\frac{\mathrm{m}}{\mathrm{m}^{\prime}}
$$

où $\mathrm{m}$ et $\mathrm{m}^{\prime}$ peuvent être exprimés en poids. $\Delta$ devient:

$$
\Delta=\mathrm{k} \frac{\mathrm{m}}{\mathrm{m}^{\prime}} \cdot \frac{1}{\mathrm{M}}
$$

$\mathrm{Si}$ on dissout $\mathrm{N}$ molécules de soluté dans $1.000 \mathrm{~g}$ de solvant, la concentration devient :

$$
\mathrm{C}=\frac{\mathrm{N} \cdot \mathrm{M}}{1.000}
$$

$\mathrm{N}$ : nombre de molécules du soluté $/ 1.000 \mathrm{~g}$ de solvant;

M : masse moléculaire du soluté.

Donc

$$
\Delta=\mathrm{k} \cdot \frac{\mathrm{N} . \mathrm{M} .}{1.000} \cdot \frac{1}{\mathrm{M}}=\mathrm{k} \cdot \frac{\mathrm{N}}{1.000}
$$

On en déduit que $\Delta$ est le même pour des solutions équimolaires ( $\mathrm{N}$ est le même).

$$
\mu=\frac{\mathrm{N}}{1.000} \text { est la molarité de la solution, }
$$

ici en molécule-gramme par $\mathrm{ml}$ de solution. Pour l'eau comme solvant, on assimile $1.000 \mathrm{~g}$ à $1.000 \mathrm{ml}$ et alors $\mu$ la molarité peut s'exprimer en moles $/ 1$ de solution, tandis que la molalité est le nombre de moles $/ \mathrm{kg}$ de solvant.

On a

$$
\Delta=\mathbf{k} \cdot \mu
$$

c) Valeurs de $\mathbf{k}$

Cette constante dépend du solvant et des unités choisies.

Dans l'eau $\Delta$ vaut $1^{\circ} 858$ pour une solution molaire non dissociée ( 1 molécule/1).

$$
\text { Quand } \mathrm{N}=1 \text {, on } \mathrm{a}: 1^{\circ} 858=\mathrm{k} \frac{1}{1.000}
$$
d'où $\mathrm{k}=1.858$ avec ces unités.

On voit aussi que $\mathrm{k}=\Delta$, si $\mathrm{C}=\mathrm{M}$ (voir $\mathrm{I}$ ), il y aurait une molécule de corps dissous par gramme de solvant mais la solution ne serait plus étendue.

Pour une solution aqueusc diluée et un soluté non électrolyte, on a :

$$
\Delta=1.858 \mu
$$

en arrondissant la valeur de $\mathrm{k}$ à 1.860 et en remplaçant $\mu$ par sa valeur $\frac{\mathrm{N}}{1.000}$

$$
\Delta=1,86 \mathrm{~N}
$$

avec $\mathrm{N}$ en molécules/1.

\section{Ecarts à la loi}

a) Explicables par $\mathrm{k}$

La constante $k$ varie quand on quitte les solutions infiniment diluées. Pour un même solvant, c'est l'ordonnée à l'origine, valeur de $\mathrm{k}$ quand $\Delta=0$, qui est constante (figure 3 ). En 


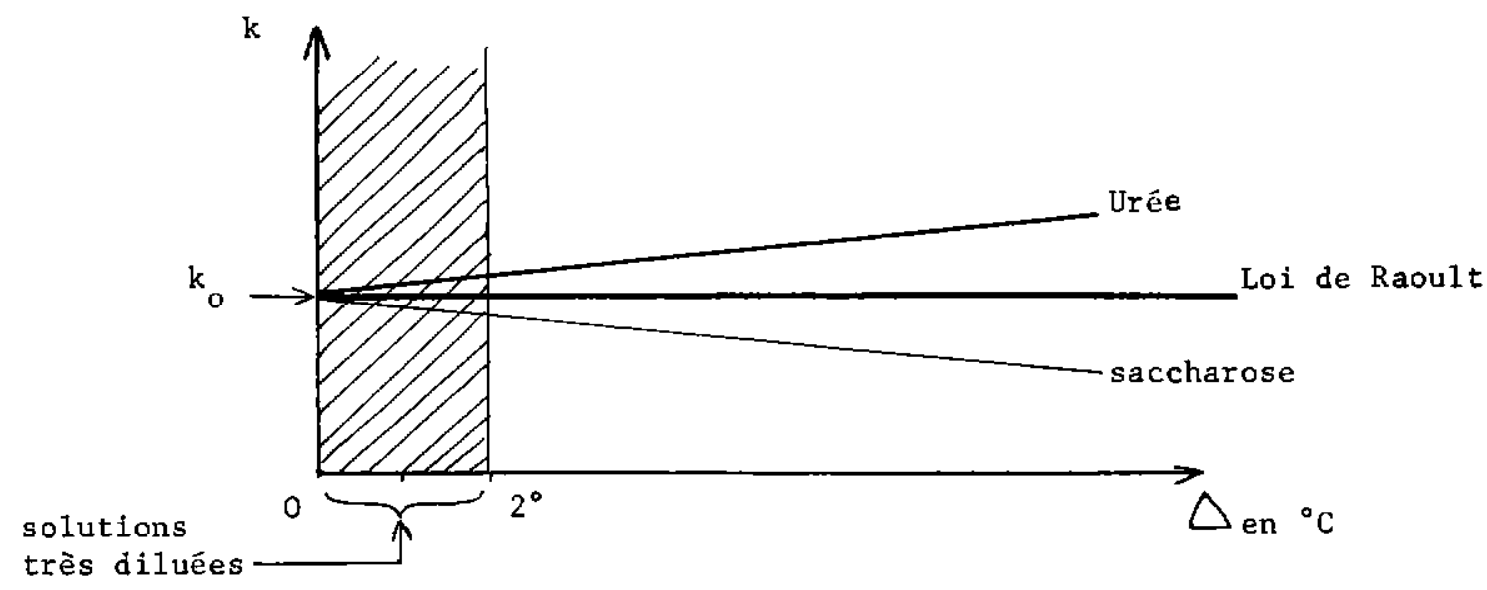

Fig. 3.

Variations de $\mathbf{k}$ pour l'urée et le saccharose en solution aqueuse. $\mathbf{k}_{0}$ représente idéalement l'expression de la loi de Raoult.

pratique, il ne faudra pas dépasser pour valeurs de $\Delta$ de $1^{\circ}$ à $2^{\circ} \mathrm{C}$ pour obtenir des résultats utilisables.

b) Cas des électrolytes eri solution dans l'eau

Il y a dissociation en ions. Prenons $N$ molécules pouvant chacune donner $\mathrm{n}$ ions. $\alpha$ étant le coefficient de dissociation, au lieu des $\mathrm{N}$ molécules on a en solution :

$\alpha \mathrm{N}$ molécules dissociées, donc $\mathrm{n} \alpha \mathrm{N}$ ions, et $(\mathrm{N}-\alpha \mathrm{N})$ molécules non dissociées, donc $(\mathrm{N}-\alpha \mathrm{N})$ molécules.

Arrhénius a montré que les ions jouent le même rôle que les molécules au point de vue de la cryométrie, au lieu de $\mathrm{N}$ molécules on a un nombre de particules:

$$
\mathrm{N}-\alpha \mathrm{N}+\mathrm{Nn} \alpha=\mathrm{N}(1+\alpha \mathrm{n}-\alpha)
$$

Le coefficient de Van 'T Hoff, rapport du nombre total de particules de la solution dissociée à celui de la solution non dissociée, est :

$$
\begin{aligned}
& \mathbf{i}=\frac{\mathrm{N}(1+\alpha \mathbf{n}-\alpha)}{\mathrm{N}} \\
& \mathbf{i}=\alpha(\mathrm{n}-1)+1
\end{aligned}
$$

Il faut donc multiplier la concentration molaire par i pour obtenir en quelque sorte la concentration particulaire, et on aura la formule générale :

$$
\Delta=\mathrm{i} \mathrm{k} \frac{\mathrm{m}}{\mathrm{m}^{\prime}} \frac{1}{\mathrm{M}} 1.000
$$

ou

$$
\Delta=\mathrm{i} \mathbf{k} \mu
$$

avec $\mathrm{i}=1$, si $\alpha=0$ dans le cas des molécules organiques non électrolytes, des composés macromoléculaires non dissociés, et des liquides biologiques.

\section{LOIS RELATIVES AUX PRESSIONS OSMOTIQUES}

Pour mémoire, nous rappelons les expériences de Dutrochet et Pfeiffer décrites précédemment. C'est Van'T Hoff principalement qui a effectué des recherches théoriques à ce sujet en appliquant les principes de la thermodynamique.

\section{Loi de Van'T Hoff}

Elle exprime l'analogie entre les mélanges gazeux, auxquels on peut appliquer la loi de Mariotte, et les solutions; elle s'énonce :

La pression qu'un corps dissous exercerait s'il était à l'état gazeux dans le même volume et à la même température, est la même que sa pression osmotique en solution.

A nouveau cette loi n'est vérifiable que pour des solutions idéales qui doivent remplir deux conditions :

$1^{0}$ être très étendues; 
$2^{\circ}$ il ne doit y avoir aucune interaction entre le solvant et le soluté.

On a donc $\pi \mathrm{V}=\pi^{\prime} \mathrm{V}^{\prime}=\mathrm{RT}$ pour des solutions " parfaites ", comme on a $\mathrm{PV}=\mathrm{P}^{\prime} \mathrm{V}^{\prime}=$ RT pour les gaz " parfaits».

$\pi$ : pression osmotique en atmosphère;

$\mathrm{V}$ : volume en litre occupé par chaque mole de soluté dans la solution à la pression osmotique $\pi, \mathrm{V}^{\prime}$ pour $\pi^{\prime}$;

$R$ : constantes des gaz parfaits exprimées en unités convenables;

$\mathrm{T}$ : température absolue, $\mathrm{T}=273,16+\mathrm{t}^{\circ} \mathrm{C}$.

\section{CONSEQUENCES DE CES LOIS. CALCUL DE LA PRESSION OSMOTIQUE}

Les conditions de validité de la loi Van'T Hoff sont les mêmes que celles énoncées avec la loi de Raoult. La méthode cryométrique de détermination des pressions osmotiques sera donc extrêmement approchée et valable seulement si on s'entoure de nombreuses précautions quant à l'interprétation des résultats.

En supposant que l'on soit dans les conditions requises, le raisonnement suivant pourra être tenu pour exprimer $\pi$ en fonction de $\Delta$ :

Pour les gaz, on a :

$$
\mathrm{PV}=\mathrm{P}^{\prime} \mathrm{V}^{\prime}=\mathrm{RT}
$$

$P$ : pression en atmosphère;

$V$ : volume en litre;

$\mathrm{T}:$ température absolue $=273,16+\mathrm{t}^{\circ} \mathrm{C}$;

$\mathrm{R}$ : constante des gaz parfaits $0,0820 \mathrm{l} /$ at ou selon l'échelle $1961\left(^{*}\right)$ :

$8,205 \times 10^{-2} 1 /$ at. molécules ${ }^{-1}$ degré $^{-1}$.

On dira qu'à $0^{\circ} \mathrm{C}$ sous une pression d'une atmosphère une mole d'un gaz occupe 22,4 1 .

Pour les solutions, on a (loi de Van 'T Hoff) :

$$
\pi \mathrm{V}=\pi^{\prime} \mathrm{V}^{\prime}=\mathrm{RT}
$$

$\pi$ : pression osmotique en atmosphère;

$\mathrm{V}$ : déjà défini s'exprime en 1 par mole. Si on assimile 11 d'eau à $1.000 \mathrm{~g}$ on aura la

(*) Toutes les constantes utilisées ici sont calculées en prenant comme référence l'échelle 1961, date à laquelle on a choisi l'isotope du carbone de poids atomique 12,000 comme étalon. relation simple $\mathrm{V}=\frac{1}{\mu}=\frac{1}{\mathrm{~N}}$

$\mathrm{N}$ devenant le nombre de moles/1 de solvant.

ou

$$
\frac{\pi}{\mathrm{N}}=\frac{\pi}{\mathrm{N}^{\prime}}=\mathrm{RT}
$$

pour des solutions aqueuses.

On dira qu'à $0^{\circ}$ sous une pression osmotique d'une atmosphère, une mole de soluté occupe 22,411 de solvant, soit $\pi V=22,41$.

Cherchons la valeur de $\pi^{\prime}$ pour une mole occupant 11 de solution, on a :

$$
1 \times 22,41=\pi^{\prime} \times 1
$$

$\pi^{\prime}: 22,41 \mathrm{~atm}$.

Autrement dit, une solution contenant 1 mole/l a une pression osmotique de 22,41 atm.

Pour cette solution, la loi de Raoult indique que $\Delta=1^{\circ} 858$ arrondi à $1^{\circ} 86$ quelle que soit la nature du soluté pourvu qu'il ne soit pas électrolyte, qu'il soit sans interaction avec le solvant, et que la concentration soit très faible, ce qui a été supposé.

Donc quand $\Delta=1^{\circ} 86$, $\pi$ vaut $22,41 \mathrm{~atm}$.;

$$
\text { si } \Delta=1^{\circ} \text {, } \pi \text { vaut } \frac{22,41}{1,86}=12,048 \mathrm{~atm} \text {. }
$$

On écrira $\pi_{0}=12,05 \Delta$ à $0^{\circ} \mathrm{C}$

D'une manière plus générale pour une solutions aqueuse :

$$
\begin{aligned}
& \Delta=1,86 \longrightarrow \pi=\mathrm{RT} \\
& \Delta=1^{\circ} \longrightarrow \pi=\frac{\mathrm{RT}}{1,86} \\
& \pi=\frac{\mathrm{RT}}{1,86} \Delta
\end{aligned}
$$

Pour les solutions dans l'eau à $t$ degré $C$ :

$$
\begin{aligned}
& \pi_{\mathrm{t}}=\frac{\mathrm{R}}{1,86}(273,16+\mathrm{t}) \Delta \\
& \pi_{\mathrm{t}}=0,441(273,16+\mathrm{t}) \Delta
\end{aligned}
$$

Ces deux dernières formules seront utilisées (VIII et IX) pour les calculs relatifs aux liquides biologiques dont on détermine $\Delta$. Dans tous les raisonnements précédents qui utilisaient 
$\mathrm{N}$ nombre de molécule/l de solution, on a abouti à la formule IV. En pratique $N$ s'y exprime en nombre de molécule $\mathrm{g} / \mathrm{l}$.

On supposera donc que les liquides biologiques sont de simples solutions aqueuses d'un soluté imaginaire; celui-ci aurait seul. même effet cryoscopique que le mélange complexe qu'on étudie en réalité.

\section{APPLICATIONS : CRYOMETRIE}

Il s'agit maintenant d'exposer quelques détails pratiques concernant la mesure indirecte de $\pi$ au moyen de l'appareil le plus simple possible.

\section{Mesure de $\Delta$ dans le cas des liquides biologiques}

\section{a) Osmole et milliosmole (osM et mosM)}

Pour les liquides biologiques, on utilise souvent le terme d'osmoles et de milliosmoles (mosM). On dénomme ainsi des particules osmotiquement actives qui sont constituées de molécules non dissociées ou d'ions.

On appelle osmole la masse de ces particules exprimée en $\mathrm{g}$.

Pour l'urée 1 osmole vaut 60 g d'urée.

Pour $\mathrm{NaCl}$ on ne pourra définir que les osmoles de $\mathrm{Na}^{+}$et de $\mathrm{Cl}^{-}$puisqu'en solution il y a dissociation de la molécule :

Pour $\mathrm{Na}+1$ osmole vaut $23 \mathrm{~g}$ de $\mathrm{Na}+$

Pour $\mathrm{Cl}^{-} 1$ osmole vaut $35,5{\mathrm{~g} \mathrm{de} \mathrm{Cl}^{-}}^{-}$

Ainsi toutes les solutions contenant une osmole/unité de volume auront même pression osmotique, cette notion permet de connaître le nombre de particules osmotiquement actives d'une solution par les poids de substances dissoutes et les lois de dissociation. Ce nombre s'obtient en divisant les poids des solutés par celui de l'osmole correspondante.

Ainsi dans 1 litre de solution $0,5 \mathrm{M}$ de $\mathrm{NaCl}$, où la dissociation en ions est pratiquement complète ( $\alpha=1$ et $\mathrm{i}=2$ ), il y a $29,25 \mathrm{~g}$ de $\mathrm{NaCl}$ dont 11,5 de $\mathrm{Na}^{+}$et 17,75 de $\mathrm{Cl}^{-}$soit en mosM :

$$
\begin{aligned}
& \frac{11,5 \times 1.000}{23}=500 \operatorname{mos} \mathrm{M} \mathrm{de} \mathrm{Na}^{+} \\
& \frac{29,25 \times 1.000}{35,5}=500 \operatorname{mos} \mathrm{M} \mathrm{de}^{-} \mathrm{Cl}^{-}
\end{aligned}
$$

On pourra dire que cette solution contient 1 osmole.

\section{b) Matériel de mesure}

Le matériel le plus simple consiste en un cryomètre de Beckmann à thermomètre (figure 4) parfois aussi nommé ultracryomètre.

Un tube à essais $\mathrm{A}$ contient la solution à étudier dans laquelle plonge un thermomètre de précision $\left(1 / 100^{\circ} \mathrm{C}\right.$ échelle de -2 à +2 par exemple) dont le réservoir est entouré des spires d'un agitateur métallique. Autour il y a un tube à essais plus grand, B, ménageant ainsi un manchon d'air périphérique régulateur de température.

L'ensemble $\mathrm{A}+\mathrm{B}$ plonge dans un mélange réfrigérant contenu dans un bécher $\mathrm{C}$.

Pour pouvoir effectuer plusicurs mesures au cours d'une journée sans avoir à rajouter trop souvent de mélange réfrigérant, on entoure $\mathbf{C}$ d'un bon isolant provenant en général d'emballages (mousses de polystyrène expansé).

\section{c) Principes des mesures}

On refroidit la solution jusqu'au moment du début de sa congélation. Pendant toute la durée de coexistence des deux phases liquide et solide la température reste constante: c'est $t$ qui est représentée par un plateau sur le graphique des températures (figure 5), en fait ce cas est rare. En général on observe le phénomène de surfusion, le liquide descend progressivement sous son point de congélation sans se solidifier (figure 5). Ce phénomène cesse parfois spontanément, pratiquement on doit le faire cesser en ajoutant quelques cristaux de solvant pur, ici de glace, à la solution dès qu'on pense être $0,5^{\circ}$ en dessous de t.

D'autre part, le plateau est très court car les $1^{\text {prs }}$ cristaux qui se forment sont riches en solvant d'où concentration de la solution restante. On notera donc le point de congélation commençante, car dès après le début de la solidification, la température baisse à nouveau régulièrement.

\section{d) Protocole d'une mesure courante}

Les solutions biologiques sont des solutions 


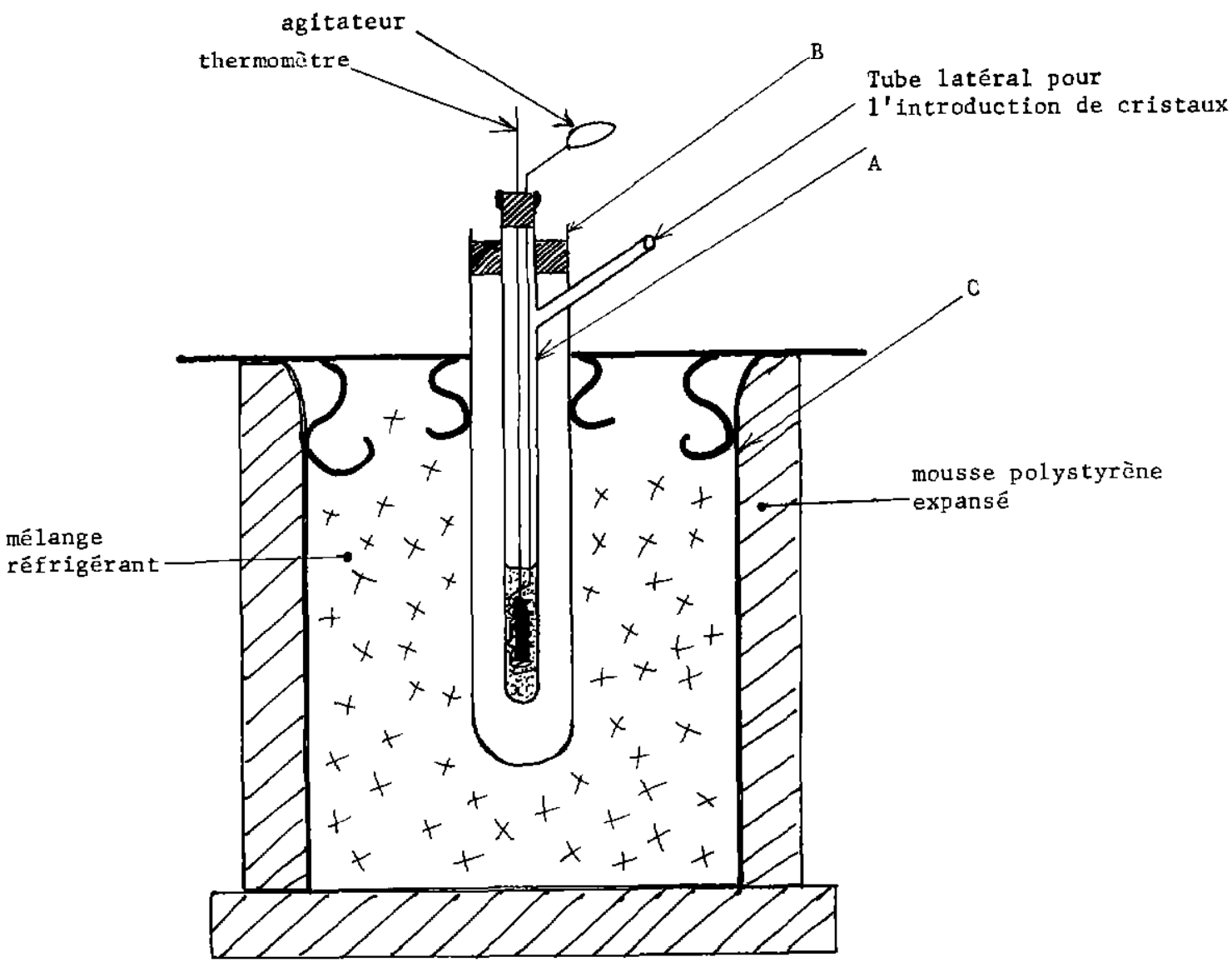

Fig. 4.

Schéma d'un cryomètre simple.

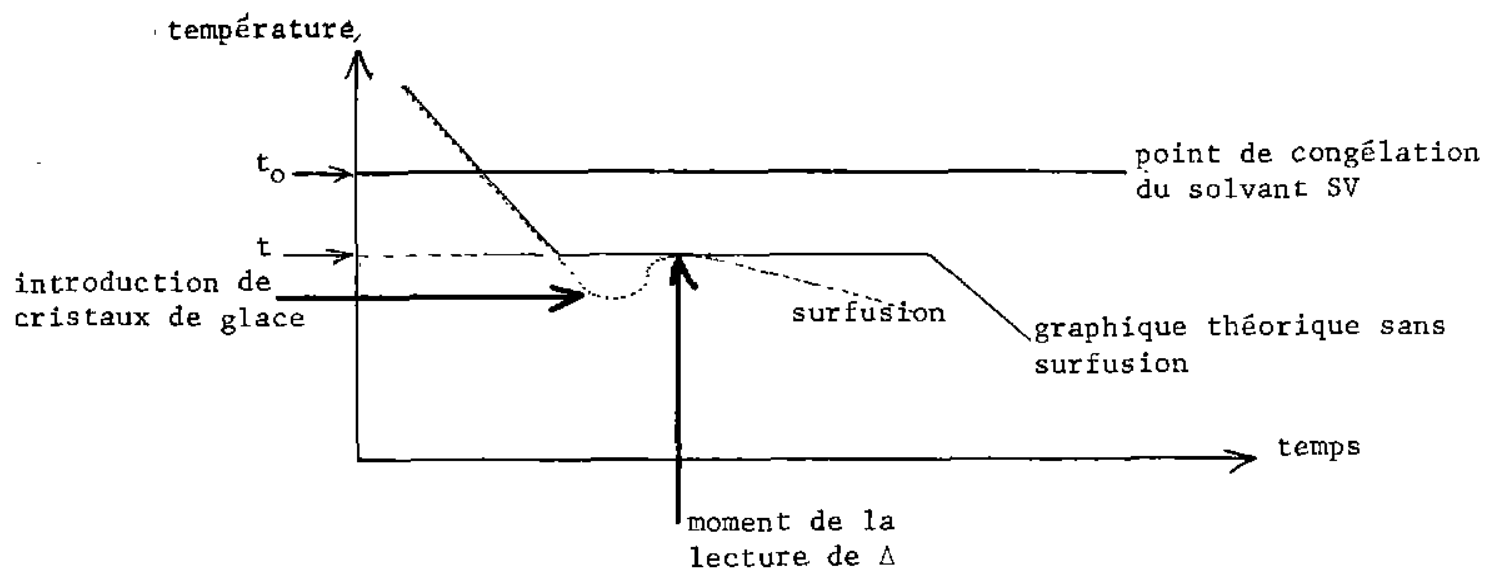

Fig. 5.

Graphique des températures théoriques avec un plateau bien horizontal et graphique réel avec le phénomène de surfusion. 
complexes dans l'eau. On considère donc $t_{0}$ $=0$.

On pourra utiliser le mélange glace pilée $\mathrm{NaCl}(5: 1)$ qui descend jusque vers $-10^{\circ} \mathrm{C}$.

Le matériel étant mis en place, on casse les cubes de glace pour en faire des fragments faciles à mélanger avec $\mathrm{NaCl}$.

Pour des mesures très fines, on utilise le mélange glace pilée $\mathrm{NO}_{3} \mathrm{~K}\left(-3^{\circ} \mathrm{C}\right)$.

\section{Etalonnage du thermomètre}

Il s'agit de faire une première expérience avec de l'eau distillée pour vérifier le $0 \mathrm{du}$ thermomètre, sachant que $\mathrm{t}^{\circ}=0^{\circ} \mathrm{C}$. $\mathrm{Si}$ on utilise un thermomètre différentiel (figure 6) l'expérience permet de fixer le 0 réel par rapport au point noté comme 0 sur l'échelle.

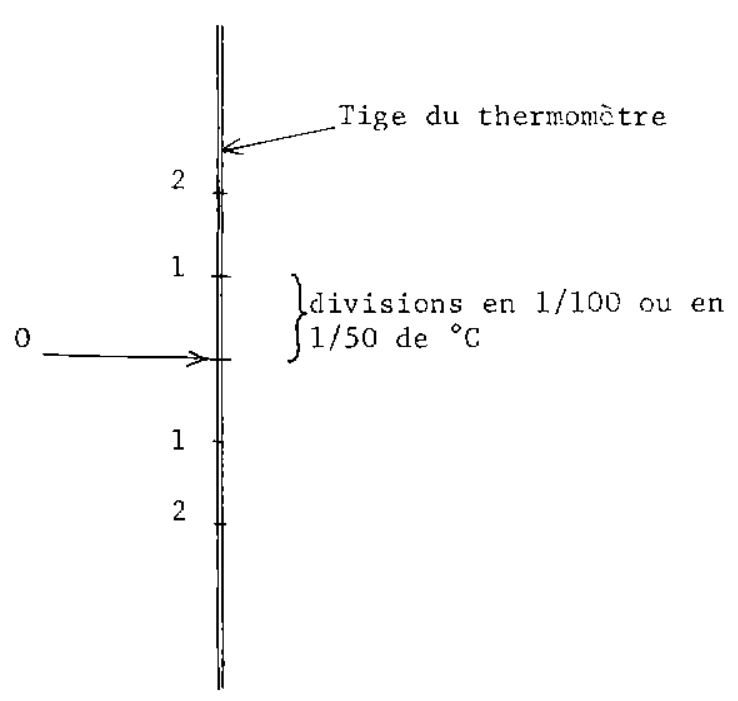

Fig. 6.

Graduations d'un thermomètre différentiel.

On verse dans le tube $A$ un volume suffisant (de 2 à $10 \mathrm{ml}$ selon les appareils), pour que le réservoir du thermomètre plonge entièrement dans le liquide et on agite régulièrement de haut en bas au rythme d'environ 120 mouvements par minute, de telle manière que les spires ne sortent pas du liquide.

On note les températures toutes les minutes, puis quand on approche $\mathrm{de}+2^{\circ} \mathrm{C}$ toutes les demi-minutes.

$\mathrm{A}-0,5^{\circ} \mathrm{C}$ on ajoute un petit cristal de glace pure, on continuera l'agitation et les mesures. On obtient le graphique des températures (figure 5) en reportant ces mesures en fonction du temps.

La graduation du thermomètre qui correspond dans le cas de l'eau à $t_{0}$ est soigneusement notée, la différence $\varepsilon$ par rapport au 0 du thermomètre ( 0 apparent) permettra de corriger la lecture de $t$ en lui retranchant $\varepsilon$ :

\section{Par exemple :}

Si $\varepsilon$ vaut 2 divisions sous le zéro apparent, $\varepsilon=-\frac{2^{\circ} \mathrm{C}}{100}$

Si $\varepsilon$ vaut 2 divisions au-dessus du zéro apparent, $\varepsilon=+\frac{2^{\circ} \mathrm{C}}{100}$

\section{Mesure de $t$}

On remplace l'eau distillée par le même volume du liquide biologique étudié, et on procède de la même manière, mais en deux étapes.

Lors de la première, on détermine $t$ approchée soit $t^{\prime}$ pour savoir quand on doit arrêter la surfusion dans l'étape qui suivra. On ajoutera donc de très petites quantités de glace pure à $-0^{\circ} 5$, si la température ne remonte pas, on opère de même à $-1^{\circ}$, et ainsi de suite à $-1^{\circ} 5$ et $-2^{\circ}$. La remontée de la température permet de déterminer $\mathbf{t}^{\prime}$.

Lors de la deuxième étape, on ajoutera les cristaux de glace à $\left(\mathrm{t}^{\prime}-0,5\right)$ et la mesure de $\mathrm{t}$ sera assez précise, le phénomène de surfusion étant modéré.

\section{e) Autre matériel pour procéder}

à des mesures indirectes de $\pi$

$\mathrm{Si}$ on utilise un dispositif à thermocouple, les opérations restent les mêmes, mais il faudra en plus étalonner le thermocouple sur des différences de températures connues avec une grande précision.

Il existe enfin d'autres appareils, très performants, qui procèdent automatiquement à l'ensemble des manipulations nécessaires à la détermination du $\Delta$, qui ont l'avantage d'être plus précis et surtout de permettre des mesures sur de faibles quantités d'échantillon : $0,2 \mathrm{ml}$ dans les cas les plus favorables.

\section{f) Résultats}

Sur 63 mesures faites sur des plasmas de 
bovins africains, on a trouvé ainsi en moyenne un $\Delta$ de $0^{\circ} 55$. On admet couramment que le sérum humain a un $\Delta$ de $0,56^{\circ}$. Il est intéressant de remarquer que ces mesures ont été faites sur des plasmas préparés à Alfort sur des échantillons de sang complet prélevés en Afrique et transportés selon le protocole mis au point pour l'étude des hémoglobines (5). Ces mesures et d'autres indiquent la parfaite conservation des échantillons.

La mesure du $\Delta$ de ces échantillons a été faite par deux méthodes : d'une part, celle qui a été longuement décrite ici, la plus simple, et d'autre part, sur un osmomètre automatique.

L'étude statistique des deux séries de résultats ( 2 mesures dans chaque cas) montre que la méthode manuelle dévie systématiquement de $+0,025^{\circ} \mathrm{C}$ en moyenne soit à $0^{\circ}$ de 0,3 atmosphère ou 6,7 mosM.

La détermination du $\Delta$ des extraits de glossines (6) montre que chez les pupes, la pression osmotique est légèrement supérieure à celle des adultes, jeunes ou vieux et aussi bien mâles que femelles (tableau II).

TABLEAD $\mathrm{N}^{\circ}$ II

\begin{tabular}{|c|c|c|c|}
\hline Espèce & $\begin{array}{c}\text { Giossina } \\
\text { morsitans morsitans }\end{array}$ & $\begin{array}{c}\text { Glosina } \\
\text { tachinoides }\end{array}$ & $\begin{array}{c}\text { Glossina } \\
\text { austeni }\end{array}$ \\
\hline Pupes de 26 jours & $691 \mathrm{mosM}$ & $540 \mathrm{mosM}$ & 471 mosM \\
\hline Jeunes or & $446 \mathrm{mosM}$ & & \\
\hline Jeunes $f$ & $528 \operatorname{mosM}$ & & \\
\hline Vieux $\sigma^{*}$ & $440 \operatorname{mos}$ & & \\
\hline
\end{tabular}

Pressions osmotiques en milliosmoles calculées d'après la mesure des $\Delta$ des hémolymphes de glossines à divers stades de leur cycle vital. Ces chiffres représentent des moyennes sur de très nombreuses mouches tsé-tsé.

\section{Mesure de $\Delta$}

\section{pour les solutions salines simples}

En particulier pour préparer des solutions isotoniques aux milieux biologiques, il est souvent nécessaire de connaître l'influence de certains sels en solution aqueuse sur la pression osmotique.

Le tableau I donne quelques informations à ce sujet pour 4 concentrations de $M / 10$ à $M$, de 26 corps. A partir du $\Delta$ on peut naturellement calculer $\pi$ en atmosphère ou en mosM.

La valeur de ces chiffres est tout à fait pratique, elle correspond aux $\Delta$ mesurables et tient donc compte des lois de la cryométrie et de la dissociation ionique. Plus la concentration est grande, plus les chances d'erreurs sont grandes. Mais on peut par une simple mesure de $\Delta$ : sur une solution simple, déterminer la molarité et le coefficient de dissociation, sur une solution complexe, leur équivalent pour une molécule hypothétique qui aurait même $\Delta$ dans les mêmes conditions.

\section{Exemples d'application}

Une fois le $\Delta$ de la solution qu'on étudie, déterminé, on peut faire trois sortes de calculs :

\section{a) Déterminer une concentration moléculaire $\mathrm{Nm}$}

On applique la formule (IV) et pour une solution aqueuse contenant une seule sorte de molécules non dissociées, on a immédiatement :

Si $\Delta=0,80$

$$
\mathrm{Nm}=\frac{\Delta}{1,86}=0,430 \text { mole-g/1 }
$$

Si la solution contient une série de corps dissociés ou non, on pourra déterminer la concentration moléculaire équivalente; ainsi pour un sérum dont $\Delta=0,56^{\circ}$.

On a $\mathrm{Nm}=\frac{0,56}{1,86}=0,301$ mole-g/l b) Calcul de $\pi$

Il a été assez insisté sur ce point pour ne pas y revenir ici. On applique la formule (VIII) si 
TABLEAU $\mathrm{N}^{\circ} \mathrm{I}$

\begin{tabular}{|c|c|c|c|c|c|}
\hline \multirow{2}{*}{$\mathrm{N} \circ \mathrm{m} \mathrm{s}$} & \multirow{2}{*}{ Formules } & \multicolumn{4}{|c|}{$\begin{array}{c}\text { Abaissemert du point de congélation } \\
\text { en degré } c: \Delta\end{array}$} \\
\hline & & $\begin{array}{l}\text { Solution } \\
\quad M / 10\end{array}$ & $M / 5$ & $M / 2$ & $M$ \\
\hline Aclde acétique & $\mathrm{CH}_{3} \mathrm{COOH}$ & 0,19 & 0,38 & 0,93 & 1,88 \\
\hline Acétone & $\mathrm{CE}_{2} \mathrm{COCH}_{3}$ & 0,19 & 0,38 & 0,93 & 1,88 \\
\hline Chlorure d'ammonium & $\mathrm{NH}_{4} \mathrm{Cl}$ & $c, 34$ & 0,71 & 1,60 & 3,42 \\
\hline Sulfate d'ammonium & $\left(\mathrm{NH}_{4}\right)_{2} \mathrm{SO}_{4}$ & 0,44 & 0,80 & 1,86 & 3,57 \\
\hline Chlorure de calcium & $\mathrm{CaCl}_{2}, 2 \mathrm{H}_{2} \mathrm{O}$ & 0,60 & $c, 98$ & 2,53 & 6,05 \\
\hline Sulfate de cuivre & $\mathrm{Cu} \mathrm{SO} 4,5 \mathrm{H}_{2} \mathrm{O}$ & 0,21 & 0,38 & 0.86 & 1,73 \\
\hline Ethano1 & $\mathrm{CH}_{3} \mathrm{CH}_{2} \mathrm{OH}$ & 0,19 & 0,38 & 0,93 & 1,89 \\
\hline D Glucose & $\mathrm{C}_{6} \mathrm{H}_{12} \mathrm{O}_{6}, 1 \mathrm{~B}_{2} \mathrm{O}$ & 0,19 & 0,38 & 1,01 & \\
\hline Glycérol & $\mathrm{CH}_{2} \mathrm{OH} \mathrm{CHOH} \mathrm{CH}_{2} \mathrm{OH}$ & 0,19 & 0,38 & 0,99 & 2,06 \\
\hline Lactose & $\mathrm{C}_{12} \mathrm{H}_{22}^{\mathrm{H}}{ }_{11}, 1 \mathrm{H}_{2} \mathrm{O}$ & 0,19 & 0,40 & & \\
\hline Chlorure de magnésium & $\mathrm{MgCl}_{2}, 6 \mathrm{H}_{2} \mathrm{O}$ & 0,53 & 0,98 & 2,68 & 6,38 \\
\hline Chlorure de potassium & KC1 & 0,34 & 0,68 & 1,69 & 3,33 \\
\hline lodure de potassium & $\mathrm{KI}$ & 0,36 & 0,71 & 1,73 & 3,57 \\
\hline Nitrate de potassium & $\mathrm{KNO}_{3}$ & 0,33 & 0.64 & 1,48 & 2,65 \\
\hline $\begin{array}{l}\text { Phosphate de potassium } \\
\text { monobasique }\end{array}$ & $\mathrm{KH}_{2} \mathrm{PO}_{4}$ & 0,33 & 0,68 & 1,53 & \\
\hline $\begin{array}{l}\text { Phosphate de potassium } \\
\text { dibasique }\end{array}$ & $\mathrm{K}_{2} \mathrm{H} \mathrm{PO}_{4}, 3 \mathrm{H}_{2} \mathrm{O}$ & 0,42 & 0,82 & & \\
\hline Sulfate de potassium & $\mathrm{K}_{2} \mathrm{SO}_{4}$ & 0,47 & 0,82 & & \\
\hline Acëtate de sodium & $\mathrm{CH}_{3} \mathrm{COONa}$ & 0,29 & 0,72 & 1,83 & 3,92 \\
\hline Bicarbonate de sodium & $\mathrm{NaH} \mathrm{CO}_{3}$ & 0,36 & 0,70 & 1,62 & \\
\hline Carbonate de sodium & $\mathrm{Na}_{2} \mathrm{CO}_{3}, 10 \mathrm{H}_{2} \mathrm{O}$ & 0,44 & 0,83 & 1,86 & \\
\hline Chlorure de sodium & $\mathrm{NaCl}$ & 0,35 & 0,70 & 1,71 & 3,45 \\
\hline $\begin{array}{l}\text { Phosphate de sodium } \\
\text { dibasique }\end{array}$ & $\mathrm{Na}_{2} \mathrm{H} \mathrm{PO}_{4}, 7 \mathrm{H}_{2} \mathrm{O}$ & 0,45 & & & \\
\hline Sulfate de sodium & $\mathrm{Na}_{2} \mathrm{SO}_{4}, 10 \mathrm{H}_{2} \mathrm{O}$ & 0,46 & 0,78 & & \\
\hline Tartrate de sodium & $\mathrm{Na}_{2}(\mathrm{COO} \mathrm{CHOH})_{2}, 2 \mathrm{H}_{2} \mathrm{O}$ & 0,45 & 0,83 & & \\
\hline Saccharose & $\mathrm{C}_{12} \mathrm{H}_{22} \mathrm{O}_{11}$ & 0,19 & 0,39 & 1,10 & 2,65 \\
\hline Urée & $\mathrm{NH}_{2} \mathrm{CONH}_{2}$ & 0,13 & 0,36 & 0,92 & 1,87 \\
\hline
\end{tabular}

Abaissement réel du point de congélation $\Delta$ en degrés centigrades pour quatre concentrations de quelques substances chimiques d'un emploi constant dans les laboratojres de biologie. Ces données tiennent compte du coefficient de dissociation des molécules de soluté (ST) en solutions aqueuses. Les cases vides correspondent à des conditions spéciales incompatibles avec les lois fondamentales qu'il faut appliquer. 
on ramène à $0^{\circ} \mathrm{C}$, la formule (IX) à la température t. Rappelons ici que la conversion des pressions osmotiques exprimées en atmosphère, en osmoles, se fait par la formule :

$$
\pi \text { at. }=\pi \text { os } \mathrm{M} \times 22,41\left(\text { à } 0^{\circ} \mathrm{C}\right) \text {. }
$$

\section{c) Détermination de $\alpha$}

Pour un sel simple en solution dans l'eau, la connaissance de $\Delta$, de $\mathrm{n}$ et de $\mathrm{M}$ permet de calculer i puis $\alpha$.

On obtient ainsi le coefficient de dissociation d'un sel dans les conditions qui ont présidées à la mesure de $\Delta$.

En conclusion, une détermination relativement simple, celle de $\Delta$ permet d'atteindre des données moléculaires intéressantes, pourvu qu'on s'entoure de toutes les précautions d'interprétation nécessaires.

\section{SYMBOLES UTILISES}

$\alpha \quad$ : coefficient de dissociation d'une solution $=$ nombre de molécules dissociées/nombre total de molécules.

C : concentration du soluté dans une solution $=$ $\frac{\mathbf{m}}{\mathbf{m}+\mathbf{m}^{\prime}}$

$\triangle \mathrm{HT}$ : pression hydrostatique.

$\Delta \quad$ : abaissement du point de congélation d'une solution, par rapport au point de congélation du solvant pur.

i : coefficient de Van'T Hoff $=$ nombre réel de particules de la solution dissociée/nombre réel de particules de la solution non dissociée. k : coefficient cryométrique, constante liée au solvant et aux unités.

m : masse du corps dissous (de soluté).

$\mathrm{m}^{\prime} \quad$ : masse du solvant correspondant à $\mathrm{m}$.

$\mu \quad$ : molarité d'une solution $=$ concentration moléculaire du soluté $=$ concentration en moles/1 de solution; la molarité d'une solution c'est le nombre de molécules-grammes par kg de solvant donc ici d'eau.

M : masse moléculaire.

mole : unité chimique de masse en C.G.S. = poids moléculaires $X$ gramme; aussi appelée molécule-gramme.

$\mathrm{N}$ : nombre de molécules de soluté dans $1.000 \mathrm{~g}$ de solvant.

$\mathrm{Nm}$ : concentration moléculaire.

n : nombre d'ions donné par une molécule.

P : pression d'un gaz (loi de Mariotte).

$\pi$ : pression osmotique en atmosphère ou en osmoles (osM).

R : constante universelle des gaz parfaits, échelle $1961=8,025 \times 10^{-2} 1 /$ at $\left(\right.$ moles$\left.^{-1}\right)$ (degrés-1).

$\mathrm{SN} \quad:$ solution $=\mathrm{SV}+\mathrm{ST}$.

ST : soluté, corps dissous.

SV : solvant.

$t_{0} \quad$ : point de congélation du solvant pur en degrés $\mathrm{C}$.

$\mathrm{t}$ : point de congélation de la solution en degrés $\mathrm{C}$.

$\mathrm{T}$ : température absolue, $\mathrm{T}=273,16+\mathrm{t}^{\mathrm{o}} \mathrm{C}$.

$\mathrm{V}, \mathrm{V}^{\prime}$ : volume de $\mathrm{SN}$ en 1 occupé par chaque molécule-g. de ST ou volume accupé par un gaz (loi de Mariotte).

$V_{0}$ : volume spécifique normal du gaz parfait, échelle $1961=2,24135 \times 10^{4} \mathrm{~cm}^{3}$ mole-1 $^{-1}$ $(22,41$ 1/mole $)$.

\section{SUMMARY}

\section{Theory and practice of the osmetic pressure measures by cryometry}

During the studies on the glossina haemolymph, the first determination concerned the osmotic pressure. The study of the animal serums cannot avoid this analytical stage and the knowledge of the saline solutions used in physiopathology also passes through determining the freezing point depression. And there are always questions concerning the calculation of the osmotic pressure, which importance in biology and variety of formulations gave way to a settlement relying on solid physical bases.

After reexposing the definitions, the theorical part of the statement allows us to pass in review both the laws concerning the molecular masses in the general case as well as in the particular cases, and the laws concerning the osmotic pressures. This leads us logically to a series of formulas that allow us to envisage practical applications, essentially the calculation of the osmotical pressure by measuring the freezing point depression of an aqueous solution. We have taken for value some constants already used and for units the constants recently retained by the international agreements. All through the establishment of the formulas, their conditions of validity are strongly underlined, then, after having taken these elementary precautions, some applications are studied in a practical point of view. 
In particular, the simplest manipulation to determine a $\Delta$ is exposed, with, for equipment, a cryometer of the Beckmann type with a differential thermometer. The technique is described step by step, which allows us to make a new measure with these only indications. The other techniques, more complicated or more expensive, are only mentioned.

A few results illustrate the whole of it; they concern the african cattle plasma and the glossina haemolymph. Lastly, a picture shows together the $\Delta$ of a few substances frequently used in laboratories; these data having been calculated or measured can save us from using a measure for which we are not always equipped.

The, whole of the symbols which are used and their meaning are shown at the end of the text to make it lighter and avoid the repetitions.

\section{RESUMEN}

\section{Teoria y práctica de las medidas de la presión osmótica mediante} criometria

Durante los estudios de la hemolinfa de las glosinas, fue la primera determinación la de la presión osmótica. Para estudiar los sueros animales se necesita tambien hacer dicha analisis, y el conocimiento de las soluciones salinas utilizadas en fisiopatologia igualmente se realiza por la determinación de los descensos del punto de congelación. Siempre se plantean cuestiones sobre el cálculo de la presión osmótica cuya importancia en biologia y cuya variedad de las formulas necesitaban una determinación según bases fisicas sólidas.

Despues de haber dado las definiciones, se pasan en revista las leyes concernientes a las masas moleculares en el caso general asi como en los casos particulares, y las concernentes a las presiones osmóticas. Se obtiene asi una serie de formulas que permite considerar aplicaciones prácticas, principalmente el cálculo de la presión osmótica al medir el descenso del punto de congelación de una solución acuosa. El valor de las constantes utilizadas y las unidades son las adoptatas más recientemente por los acuerdos internacionales. Durante el establecimiento de las formulas, se notan sus condiciones de validez; Luego se estudian las aplicaciones prácticas. Se expone particularmente la manipulación más simple para determinar un $\Delta$, con un criometro de tipo Beckmann con termometro diferencial. Se describe en detalle la tecnica, lo que permite hacer de nuevo una medida siguiendo dichas solas indicaciones. Solo se mencionan otras tecnicas más complejas y más costosas.

Se dan algunos resultados que conciernen los plasmas de bovinos africanos y las hemolinfas de glosinas. Un cuadro reune los $\Delta$ de algunas substancias corrientemente utilizadas en el laboratorio, estos datos, calculados o medidos, pueden evitar de hacer una medida por la cual no se tiene siempre el aparato.

Se indican la totalidad de los símbolos utilizados y su significación a fin del texto para evitar las repiticiones.

\section{BIBLIOGRAPHIE}

I. BENEZECH (C.), «Physicochimie biologique et médicale », Paris, Masson et Cie, 1958, pp. 177188.

2. GEIGY (S.A.), (Departement pharmaceutique) "Tables scientifiques », 6e éd., Bâle, J. R. GEIGY. 1963, pp. 330-337.

3. LOISELEUR (J.), "Techniques de laboratoire», t. I, 1er fasc., Paris, Masson et Cie, 1963, pp. 68-71.

4. OLIVIER (H. R.), "Traité de biologie pratique », t. I, Paris, Maloine, 1961, pp. 235-240.
5. PETIT (J. P.), « Protocole de récolte et de transport de sang pour l'étude comparée des hémoglobines de taurin et de zébu. Valeur des échantillons ansi obtenus », Compte-rendu du Symposium International sur la structure comparée des hémoglobines, Thessalonique 11-13 avril 1966 pp. $122-125$.

6. PETIT (J.P.), « Hémolymphe de glossines: récolte et analyse ", Rev. Elev. Méd. vét. Pays trop., 1968, 21 (4) : 493-497. 